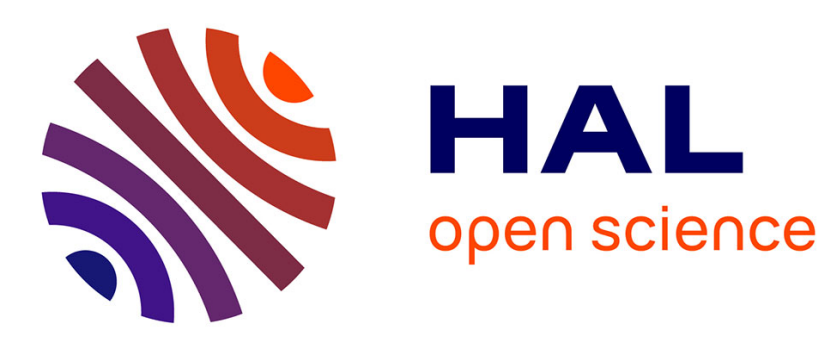

\title{
Observability analysis for structured bilinear systems: a graph-theoretic approach
}

Taha Boukhobza, Frédéric Hamelin

\section{To cite this version:}

Taha Boukhobza, Frédéric Hamelin. Observability analysis for structured bilinear systems: a graphtheoretic approach. Automatica, 2007, 43 (11), pp.1968-1974. 10.1016/j.automatica.2007.03.010 . hal-00159428

\section{HAL Id: hal-00159428 \\ https://hal.science/hal-00159428}

Submitted on 3 Jul 2007

HAL is a multi-disciplinary open access archive for the deposit and dissemination of scientific research documents, whether they are published or not. The documents may come from teaching and research institutions in France or abroad, or from public or private research centers.
L'archive ouverte pluridisciplinaire HAL, est destinée au dépôt et à la diffusion de documents scientifiques de niveau recherche, publiés ou non, émanant des établissements d'enseignement et de recherche français ou étrangers, des laboratoires publics ou privés. 


\title{
Observability analysis for structured bilinear systems: a graph-theoretic approach
}

\author{
T. Boukhobza and F. Hamelin \\ Centre de Recherche en Automatique de Nancy (CRAN - CNRS UMR 7039), Nancy University, \\ BP 239, 54506 Vandouvre Cedex, Nancy, France, \\ Phone: 33383684 464, Fax: 33383684 462, email: taha.boukhobza@cran.uhp-nancy.fr
}

\begin{abstract}
This paper is devoted to the generic observability analysis for structured bilinear systems using a graph-theoretic approach. On the basis of a digraph representation, we express in graphic terms the necessary and sufficient conditions for the generic observability of structured bilinear systems. These conditions have an intuitive interpretation and are easy to check by hand for small systems and by means of well-known combinatorial techniques for large scale systems.
\end{abstract}

Key words: Structured bilinear systems, generic observability, graph theory.

\section{Introduction}

The class of bilinear systems (BLS), representing particular nonlinear systems whose dynamics are jointly linear in the state and the input variables, was introduced in control theory in the 1960's. This kind of systems are simpler and better understood than most other nonlinear systems. Furthermore, industrial process control, economics and biology (switched circuits, mechanical brakes, controlled suspension systems, immunological systems, population growth, enzyme kinetics, ...) provide examples of BLS. Finally, the usual linearization of a nonlinear control system near an equilibrium point can be improved by using a bilinear approximation. For these reasons, many works deal with BLS.

The observability of BLS was tackled in many works among which we can cite $[7,18]$. The necessary and sufficient conditions to achieve this property are now very well known. These conditions have been established using essentially geometric or algebraic tools. However, the use of such tools assumes the exact knowledge of the state space matrices characterizing the system's model. In many modeling problems, these matrices have a number of fixed zero entries determined by the physical laws while the remaining entries are not known precisely. To study the properties of these systems in spite of the poor knowledge we have on them, the idea is that we only keep the zero/non-zero entries in the state space matrices. Thus, we consider models where the fixed zeros are conserved while the non-zero entries are replaced by free parameters. There is a huge amount of interesting works in the literature using this kind of models called structured models. These models are useful to describe the class of systems having the same structure and they capture most of the structural available information from physical laws. Moreover, their study requires a low computational burden which allows one to deal with large scale systems. Be- cause of these features, we think that structured systems are adapted to study a property like the observability and subsequently this paper deals with this kind of systems.

Many results on structured systems are related to the graphtheoretic approach. This approach is mainly dedicated to linear systems for which many structural properties such as controllability, observability, solvability of several classical control problems including disturbance rejection, inputoutput decoupling, fault detection and isolation are studied. Survey paper [6] reviews the most significant results in this area. From these studies, it follows that the graphtheoretic approach provides simple and elegant solutions and so is very well-suited to analyse large scale or/and uncertain systems. Unfortunately, not so many works based on graph-theoretic methods deal with nonlinear systems. Among them, $[9,10]$ give conditions to the input-output decoupling and linearization of a nonlinear system, [15] gives sufficient conditions to fulfill the observability of bilinear systems and recently, [2] provides sufficient conditions for the uniform observability of some nonlinear systems. In this context, this paper is dedicated to the analysis of the observability for structured bilinear systems (SBLS). More precisely, for such class of systems, we give simple necessary and sufficient conditions to achieve the observability of the whole or of a given part of the state. These conditions, which are equivalent to the well-known observability criteria $[7,14,18]$ are easier to check from a computational point of view.

The paper is organised as follows: after section 2, which is devoted to the problem formulation, a digraph representation of SBLS is defined in section 3. The main results are given in section 4 and are illustrated with an example in section 5. Finally, some concluding remarks are made. 


\section{Problem statement}

In this paper, we consider SBLS in the form:

$$
\left(\Sigma_{\Lambda}\right):\left\{\begin{array}{l}
\dot{x}(t)=A_{0} x(t)+\sum_{i=1}^{m} u_{i}(t) A_{i} x(t)+B u(t) \\
y(t)=C x(t)
\end{array}\right.
$$

where $x(t)=\left(x_{1}(t), \ldots, x_{n}(t)\right)^{T} \in \mathbb{R}^{n}, u(t)=$ $\left(u_{1}(t), \ldots, u_{m}(t)\right)^{T} \in \mathbb{R}^{m}$ and $y(t)=\left(y_{1}(t), \ldots, y_{p}(t)\right)^{T} \in \mathbb{R}^{p}$ are respectively the state, the input and the output vectors. For $i=0, \ldots, m, A_{i} \in \mathbb{R}^{n \times n}, B \in \mathbb{R}^{n \times m}$ and $C \in \mathbb{R}^{p \times n}$ are matrices which elements are either fixed to zero or assumed free non-zero parameters. We can parameterize these nonzero entries by scalar real (nonzero) parameters $\lambda_{i}, i=1, \ldots, h$ forming a parameter vector $\Lambda=\left(\lambda_{1}, \lambda_{2}, \ldots, \lambda_{h}\right)^{T} \in \mathbb{R}^{h}$. If all the parameters $\lambda_{i}$ are fixed, we obtain an admissible realization of structured system $\left(\Sigma_{\Lambda}\right)$. Theoretic properties of each realization can be studied according to the values of $\lambda_{i}$. We say that a property is true generically [5] if it is true for almost all the realizations of structured system $\left(\Sigma_{\Lambda}\right)$. Here, "for almost all the realizations" is to be understood $[6,17]$ as "for all parameter values $\left(\Lambda \in \mathbb{R}^{h}\right)$ except for those in some proper algebraic variety in the parameter space". The proper algebraic variety for which the property is not true is the zero set of some nontrivial polynomial with real coefficients in the $h$ system parameters, which can be written down explicitly. Recall that a proper algebraic variety is an algebraic variety which has Lebesgue measure zero.

Many studies deal with the observability of bilinear systems using generally either geometric tools or linear timevarying system theory [4]. In these studies, the notions of $u$-indistinguishability, $u$-observability and $u$-unobservability subspaces are defined to characterize the ability of state reconstruction using the knowledge of the input and the output. In this respect, observability definition of BLS is given in [18]. We extend this definition to SBLS:

Definition 1 Structured bilinear system $\left(\Sigma_{\Lambda}\right)$ is generically observable if, for almost all the realisations of $\left(\Sigma_{\Lambda}\right)$, there exists an input $u(t)$ such that any pair of initial states $x^{0}(0)$ and $x^{1}(0)$ are distinguishable by observation of the corresponding outputs $y^{0}(t)$ and $y^{1}(t)$ for $t \geq 0$.

Directly, from results provided in [18], the generic observability of SBLS $\left(\Sigma_{\Lambda}\right)$ is necessary and sufficient to ensure, for almost all the realizations of $\left(\Sigma_{\Lambda}\right)$, the existence of an observer which provides an asymptotic estimate of the state. Moreover, Theorem 1 of [7] states that for piecewise constant or continuous controls, a BLS is observable iff there exists an input $u(t)$ for which it is $u$-observable. The proof is based on the construction of an universal input $\bar{u}$, which distinguishes all initial states $x^{1}(0)$ from $x^{0}(0)=\mathbf{0}$ by concatenating at most $n+1$ constant inputs: $\bar{u}=u^{0} \circ u^{1} \circ \ldots \circ u^{n}$. At the $k^{\text {th }}$ stage in the construction, the set of states indistinguishable from $x(0)=\mathbf{0}$ is reduced in dimension by well chosen input value $u^{k}$. This leads to the following observability criterion for BLS. This criterion is based on the observability matrix rank conditions proposed in [7] for BLS.
Theorem 2 For piecewise continuous input signals $u(t)$, structured bilinear system $\left(\Sigma_{\Lambda}\right)$ is generically observable iff T1: g_rank $\left(O\left(C, A_{0}, A_{1}, \ldots, A_{m}\right)\right)=n$, where

$$
\begin{aligned}
& O\left(C, A_{0}, A_{1}, \ldots, A_{m}\right)=\operatorname{col}\left(C, C A_{0}, C A_{1}, \ldots, C A_{m},\right. \\
& \left.C A_{0}^{2}, C A_{0} A_{1}, \ldots \ldots, C A_{0} A_{m}, C A_{1} A_{0}, \ldots, C A_{m}^{n-1}\right)
\end{aligned}
$$

is the observability matrix of system $\left(\Sigma_{\Lambda}\right)$ and where $g_{\text {rank }}(M)$ denotes the generic rank of matrix $M$ [12].

The aim of this paper is to provide graphic conditions equivalent to the one of Theorem 2. It turns out that these graphic conditions are easier to check and so more adapted to tackle large scale or uncertain systems. Indeed, the computation of g_rank $\left(O\left(C, A_{0}, A_{1}, \ldots, A_{m}\right)\right)$ is quite difficult particularly for large scale systems.

\section{Graph representation of structured bilinear systems}

This section is devoted, in a first stage, to the definition of a digraph which represents $\operatorname{SBLS}\left(\Sigma_{\Lambda}\right)$. Next, some useful notations and definitions are given.

\subsection{Digraph definition for structured bilinear system}

Not so many works use the graph-theoretic approach to study nonlinear systems. The digraph we use in this paper is quite close to the one presented in [10]. The main differences are due to the fact that we adapt our representation to the context of observability analysis and to the fact that we deal with structured systems.

The digraph we associate to $\left(\Sigma_{\Lambda}\right)$ is noted $\mathcal{G}\left(\Sigma_{\Lambda}\right)$ and is constituted by a vertex set $\mathcal{V}$ and an edge set $\mathcal{E}$ i.e. $\mathcal{G}\left(\Sigma_{\Lambda}\right)=$ $(\mathcal{V}, \mathcal{E})$. The vertices are associated to the state and the output components of $\left(\Sigma_{\Lambda}\right)$ and the directed edges represent links between these variables.

More precisely, $\mathcal{V}=\mathbf{X} \cup \mathbf{Y}$, where $\mathbf{X}=\left\{\mathbf{x}_{\mathbf{1}}, \ldots, \mathbf{x}_{\mathbf{n}}\right\}$ is the set of state vertices, $\mathbf{Y}=\left\{\mathbf{y}_{\mathbf{1}}, \ldots, \mathbf{y}_{\mathbf{p}}\right\}$ is the set of output vertices. For sake of clarity, the state and the output vertices are written in bold fonts. The edge set is $\mathcal{E}=\bigcup_{l=0}^{m} A_{l}$-edges $\cup C$-edges, where, for $l=0, \ldots, m, A_{l}$-edges $=\left\{\left(\mathbf{x}_{\mathbf{j}}, \mathbf{x}_{\mathbf{i}}\right) \mid A_{l}(i, j) \neq 0\right\}$ and $C$-edges $=\left\{\left(\mathbf{x}_{\mathbf{j}}, \mathbf{y}_{\mathbf{i}}\right) \mid C(i, j) \neq 0\right\}$.

Here $M(i, j)$ is the $(i, j)$ th element of matrix $M$ and $\left(\mathbf{v}_{\mathbf{1}}, \mathbf{v}_{\mathbf{2}}\right)$ denotes a directed edge from vertex $\mathbf{v}_{\mathbf{1}} \in \mathcal{V}$ to vertex $\mathbf{v}_{\mathbf{2}} \in \mathcal{V}$. Note that number $i$ is indicated under each $A_{i}$-edge in order to preserve the information about the belonging of the edges in the digraph representation. Moreover, for $i \in\{0, \ldots, m\}$, to each edge $e \in A_{i}$-edges we associate index $i$. Note that we associate several indexes to an edge $e$ if it belongs to several subsets $A_{i}$-edges. The following example illustrates the proposed digraph representation.

Example 3 In Figure 1, we represent the digraph associated to the SBLS defined by: 


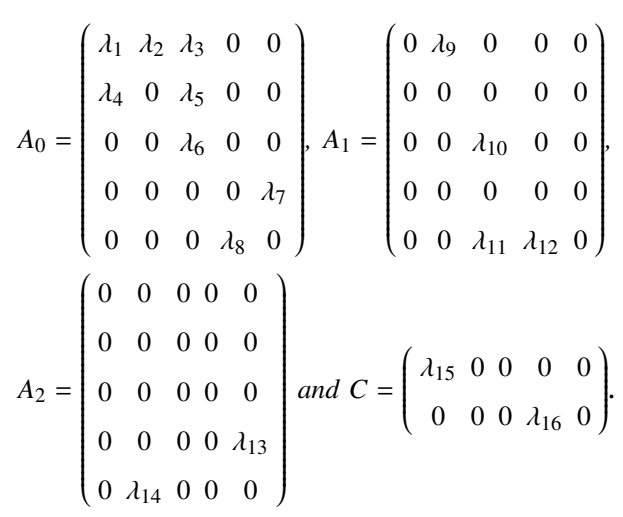

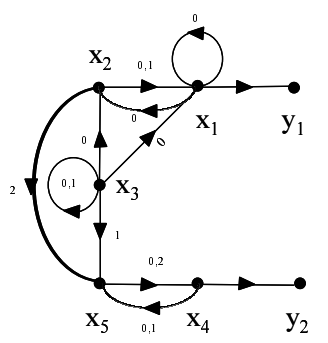

Figure 1. Digraph for the study of observability for Example 3

As we can see, comparatively with the digraph presented in [10], the input vertices are removed since we study the state observability. Moreover, due to this removal, the edges, which start in [10] from the input vertices, start in our digraph from state vertices and constitute the $A_{i}$-edges. Finally, as we are interested in structured systems, each edge represents a free parameter which has no numerical value. Thus, it is not necessary to indicate it on each edge of the digraph as in [10].

\subsection{Definitions and notations}

We present hereafter briefly some definitions concerning particular tools, we use in the paper. For the classical definitions related to the digraphs, the readers are referred to $[1,6,10,12,13]$.

- A path $P$ is an $\mathbf{Y}$-topped path if its end vertex is an element of $\mathbf{Y}$.

- Two edges $e_{1}=\left(\mathbf{v}_{\mathbf{1}}, \mathbf{v}_{\mathbf{1}}^{\prime}\right)$ and $e_{2}=\left(\mathbf{v}_{\mathbf{2}}, \mathbf{v}_{\mathbf{2}}^{\prime}\right)$, elements of $\mathcal{E}$, are $v$-disjoint if $\mathbf{v}_{\mathbf{1}} \neq \mathbf{v}_{\mathbf{2}}$ and $\mathbf{v}_{\mathbf{1}}^{\prime} \neq \mathbf{v}_{\mathbf{2}}^{\prime}$. Note that $e_{1}$ and $e_{2}$ can be $v$-disjoint even if $\mathbf{v}_{\mathbf{1}}^{\prime}=\mathbf{v}_{\mathbf{2}}$ or $\mathbf{v}_{\mathbf{1}}=\mathbf{v}_{\mathbf{2}}^{\prime}$. $k$ edges are $v$ disjoint if they are mutually $v$-disjoint.

- Consider $k$ edges $e_{1}=\left(\mathbf{v}_{\mathbf{1}}, \mathbf{v}_{\mathbf{1}}^{\prime}\right), e_{2}=\left(\mathbf{v}_{\mathbf{2}}, \mathbf{v}_{\mathbf{2}}^{\prime}\right), \ldots, e_{k}=$ $\left(\mathbf{v}_{\mathbf{k}}, \mathbf{v}_{\mathbf{k}}^{\prime}\right)$. We define for $i=1, \ldots, k, I_{i}$ as the set of integers $j$ such that $\mathbf{v}_{\mathbf{j}}^{\prime}=\mathbf{v}_{\mathbf{i}}^{\prime}$ i.e. $I_{i} \stackrel{\text { def }}{=}\left\{1 \leq j \leq k \mid \mathbf{v}_{\mathbf{j}}^{\prime}=\mathbf{v}_{\mathbf{i}}^{\prime}\right\} . e_{1}, e_{2}, \ldots$, $e_{k}$ are $A$-disjoint if $\mathbf{C} \mathbf{1}$ and $\mathbf{C 2}$ are satisfied, where:

C1: edges $e_{1}, e_{2}, \ldots, e_{k}$ have distinct begin vertices.

C2: for $i=1, \ldots, k,\left(I_{i}=\{i\}\right)$ or (there exist $r$ distinct integers $i_{1}, i_{2}, \ldots, i_{r}$ such that $e_{j_{1}} \in A_{i_{1}}$-edges, $e_{j_{2}} \in$ $A_{i_{2}}$-edges, $\ldots, e_{j_{r}} \in A_{i_{r}}$-edges, where $j_{1}, j_{2}, \ldots, j_{r}$ are all the elements of $I_{i}$ ).

Roughly speaking, $k$ edges are $A$-disjoint if their begin vertices are all distinct and if all the edges which have the same end vertex can be associated to distinct indexes $i$.
To illustrate the latter definition, note that in Example 3, $e_{1}=\left(\mathbf{x}_{2}, \mathbf{x}_{1}\right)$ and $e_{2}=\left(\mathbf{x}_{1}, \mathbf{x}_{1}\right)$ are $A$-disjoint as well as $e_{1}=\left(\mathbf{x}_{2}, \mathbf{x}_{1}\right)$ and $e_{3}=\left(\mathbf{x}_{3}, \mathbf{x}_{1}\right)$. So, $e_{1}, e_{2}$ and $e_{3}$ are mutually $A$-disjoint but they are not $A$-disjoint even if condition $\mathbf{C 1}$ is satisfied. Indeed, these three edges have the same end vertex i.e. $I_{1}=I_{2}=I_{3}=\{1,2,3\}$ and there do not exist three distinct integers $i_{1}, i_{2}$ and $i_{3}$ such that $e_{1} \in A_{i_{1}}$-edges, $e_{2} \in A_{i_{2}}$ edges and $e_{3} \in A_{i_{3}}$-edges. Thus, $\mathbf{C 2}$ is not satisfied. Otherwise, edges $e_{1}=\left(\mathbf{x}_{\mathbf{2}}, \mathbf{x}_{\mathbf{5}}\right), e_{2}=\left(\mathbf{x}_{\mathbf{3}}, \mathbf{x}_{\mathbf{5}}\right)$ and $e_{3}=\left(\mathbf{x}_{\mathbf{4}}, \mathbf{x}_{\mathbf{5}}\right)$ are $A$-disjoint. Indeed, as they have distinct begin edges, $\mathbf{C 1}$ is satisfied. Moreover, even if $I_{1}=I_{2}=I_{3}=\{1,2,3\}$, as $e_{1} \in A_{2}$-edges, $e_{2} \in A_{1}$-edges and $e_{3} \in A_{0}$-edges, $\mathbf{C 2}$ is satisfied too.

\section{Main results}

We give hereafter a graphical criterion to characterize the generic-observability of system $\left(\Sigma_{\Lambda}\right)$.

Proposition 4 Structured bilinear system $\left(\Sigma_{\Lambda}\right)$ is generically observable iff in its associated digraph $\mathcal{G}\left(\Sigma_{\Lambda}\right)$ i. every state vertex is the begin vertex of an $\mathbf{Y}$-topped path; ii. there exist $n$ A-disjoint edges in $\mathcal{G}\left(\Sigma_{\Lambda}\right)$.

\section{Proof:}

Necessity:

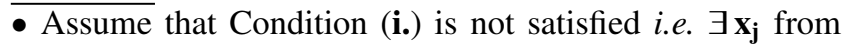
which there does not exist an Y-topped path. Then, we can state that, $\forall k>0, \forall i_{1}, i_{2}, \ldots, i_{k}$ elements of $\{0,1, \ldots, m\}$, the $j^{\text {th }}$ column of $C A_{i_{1}} A_{i_{2}} \ldots A_{i_{k}}$ is equal to zero. Thus, condition $\mathbf{T 1}$ of Theorem 2 cannot be satisfied and the system is not generically observable.

- If structured bilinear system $\left(\Sigma_{\Lambda}\right)$ is generically observable i.e. g_rank $\left(O\left(C, A_{0}, A_{1}, \ldots, A_{m}\right)\right)=n$ then $\operatorname{Im}\left(C^{T}, A_{0}^{T} C^{T}, A_{1}^{T} C^{T}, \ldots, A_{m}^{T} C^{T},\left(A_{0}^{T}\right)^{2} C^{T}\right.$, $\left.A_{0}^{T} A_{1}^{T} C^{T}, \ldots, A_{0}^{T} A_{m}^{T} C^{T}, \quad A_{1}^{T} A_{0}^{T} C^{T}, \ldots\left(A_{m}^{T}\right)^{n-1} C^{T}\right)=\mathbb{R}^{n}$. Yet, since $\forall M \in \mathbb{R}^{n \times p}, \operatorname{Im}\left(A_{i}^{T} M\right) \subseteq \operatorname{Im}\left(A_{i}^{T}\right)$, we have that $\operatorname{Im}\left(C^{T}, A_{0}^{T} C^{T}, A_{1}^{T} C^{T}, \ldots, A_{m}^{T} C^{T},\left(A_{0}^{T}\right)^{2} C^{T}, \ldots, A_{0}^{T} A_{m}^{T} C^{T}\right.$, $\left.A_{1}^{T} A_{0}^{T} C^{T}, \ldots\left(A_{m}^{T}\right)^{n-1} C^{T}\right) \subseteq \operatorname{Im}\left(C^{T}, A_{0}^{T}, \ldots, A_{m}^{T}\right) \subseteq \mathbb{R}^{n}$

Thus, necessarily $\mathrm{g} \_\operatorname{rank}\left(O\left(C, A_{0}, A_{1}, \ldots, A_{m}\right)\right)=n$ implies that $\operatorname{Im}\left(C^{T}, A_{0}^{T}, A_{1}^{T}, \ldots, A_{m}^{T}\right)=\mathbb{R}^{n}$ and so g_rank $\left(C^{T}, A_{0}^{T}, A_{1}^{T} \ldots, A_{m}^{T}\right)=n$ which is equivalent to g_rank $\left(\operatorname{col}\left(C, A_{0}, A_{1}, \ldots, A_{m}\right)\right)=n$.

On the other hand, we can prove (Appendix A) using the results of $[12,13,16]$, that:

Statement 1: The maximal number of $A$-disjoint edges in $\mathcal{G}\left(\Sigma_{\Lambda}\right)$ is equal to g_rank $\left(\operatorname{col}\left(C, A_{0}, A_{1}, \ldots, A_{m}\right)\right)$.

Thus, immediately, the generic-observability of $\operatorname{SBLS}\left(\Sigma_{\Lambda}\right)$ implies that condition (ii.) is satisfied.

Sufficiency:

$\overline{\text { At first, let }}$ us recall some facts on linear systems. Consider a structured linear system,

$$
\Sigma_{L, \Lambda}:\left\{\begin{array}{l}
\dot{x}(t)=A x(t)+B u(t) \\
y(t)=C x(t)
\end{array}\right.
$$


It is well known that the system is observable iff g_rank $\left(\begin{array}{c}C \\ s I-A\end{array}\right)=n, \forall s \in \mathbb{C}$. Otherwise, the PBH test [8] states that $\left(\Sigma_{L, \Lambda}\right)$ is unobservable iff there exists generically a vector $q \neq 0$ such that $A q=s_{0} q, s_{0} \in \mathbb{C}$ and $C q=0$.

On the one hand, if $g_{-}$rank $\left(\begin{array}{c}C \\ s I-A\end{array}\right)=n, \forall s \in \mathbb{C} \backslash\{0\}$, then the unobservability of $\left(\Sigma_{L, \Lambda}\right)$ implies necessarily that there exists generically a vector $q \neq 0$ such that $A q=0$ and $\mathrm{Cq}=0$.

On the other hand, Lemma 14.1 of [13] states that, if in the digraph associated to $\left(\Sigma_{L, \Lambda}\right)$, every state vertex is a begin vertex of an Y-topped path, then $g_{-}$rank $\left(\begin{array}{c}C \\ s I-A\end{array}\right)=n$, $\forall s \in \mathbb{C} \backslash\{0\}$.

Assume now that conditions of Proposition 4 are satisfied. On the one hand, using Lemma 14.1 of [13], as all the parameters of matrices $A_{0}, \ldots, A_{m}$ are assumed to be free, we can state that condition (i.) of Proposition 4 implies that, for almost all input values $\bar{u}=\left(\bar{u}_{1}, \ldots, \bar{u}_{m}\right)^{T}$, we have g_rank $\left(\operatorname{col}\left(C, s I-\left(A_{0}+\bar{u}_{1} A_{1}+\ldots+\bar{u}_{m} A_{m}\right)\right)\right)=n, \quad \forall s \neq 0$. On the other hand, if SBLS $\left(\Sigma_{\Lambda}\right)$ is generically unobservable, then for all input constant values, $\bar{u}=\left(\bar{u}_{1}, \ldots, \bar{u}_{m}\right)^{T}$, linear systems defined by matrices $(C, \bar{A})$ are also unobservable, where $\bar{A}=A_{0}+\sum_{i=1}^{m} \bar{u}_{i} A_{i}$. This is due to the fact that for all input constant values, $\bar{u}$, $\operatorname{Im}\left(O(C, \bar{A}) \subseteq \operatorname{Im}\left(O\left(C, A_{0}, A_{1}, \ldots, A_{m}\right)\right)\right.$.

Therefore, If $\left(\Sigma_{\Lambda}\right)$ is unobservable, using the previous settings, we have that for each matrix $\bar{A}$, there exists a nonzero vector $q$ such that $\bar{A} q=0$ and $C q=0$. Since this statement is true for almost all the input values $\bar{u}=\left(\bar{u}_{1}, \ldots, \bar{u}_{m}\right)^{T}$, we have that for almost $n(m+1)$-uple values of input noted $\bar{u}^{j}=\left(\bar{u}_{1}^{j}, \ldots, \bar{u}_{m}^{j}\right)^{T}, j=1, \ldots, n(m+1)$, we can find nonzero vectors $q_{j}$ such that the following system is satisfied:

$$
\left\{\begin{array}{c}
C q_{j}=0 \\
A_{0} q_{j}+\sum_{i=1}^{m} \bar{u}_{i}^{j} A_{i} q_{j}=0 \quad j=1, \ldots, n(m+1)
\end{array}\right.
$$

Obviously, there cannot exist more than $n$ independent vectors $q_{j}$. Let us note $q_{1}, \ldots, q_{n}$ the vectors such that $\operatorname{span}\left(q_{1}, q_{2}, \ldots, q_{n(m+1)}\right) \subseteq \operatorname{span}\left(q_{1}, q_{2}, \ldots, q_{n}\right)$ (we can renumber the vectors if necessary). All the vectors $q_{j}$, $j=n+1, \ldots, n(m+1)$ are linear combinations of $q_{1}, \ldots, q_{n}$. Therefore, system (2) contains the following $n(m+1)+n$ equations

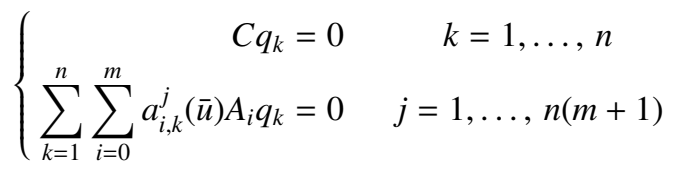

where $a_{i, k}^{j}(\bar{u})$ are linear functions of $\bar{u}^{j}, j=1, \ldots, n(m+1)$. Since system (2) is satisfied for almost all the input values, we can generically find $\bar{u}^{j}, j=1, \ldots, n(m+1)$ such that

$$
\operatorname{det}\left(\begin{array}{cccc}
a_{0,1}^{1}(\bar{u}) & a_{0,2}^{1}(\bar{u}) & \ldots & a_{m, n}^{1}(\bar{u}) \\
a_{0,1}^{2}(\bar{u}) & a_{0,2}^{2}(\bar{u}) & \ldots & a_{m, n}^{2}(\bar{u}) \\
\vdots & \vdots & \vdots & \vdots \\
a_{0,1}^{n(m+1)}(\bar{u}) & a_{0,2}^{n(m+1)}(\bar{u}) & \ldots & a_{m, n}^{n(m+1)}(\bar{u})
\end{array}\right) \neq 0 \text {. In this case, }
$$

the only solution of system (3) is

$$
\left\{\begin{array}{c}
C q_{k}=0 \\
A_{0} q_{k}=A_{1} q_{k}=\ldots=A_{m} q_{k}=0 \quad k=1, \ldots, n
\end{array}\right.
$$

Obviously, if $\left(\Sigma_{\Lambda}\right)$ is unobservable then at least one vector $q_{k}, k=1, \ldots, n$ is nonzero. Consequently, $\operatorname{SBLS}\left(\Sigma_{\Lambda}\right)$ is unobservable only if there exists at least a nonzero vector $q$ such that $C q=0$ and $A_{0} q=A_{1} q=\ldots=A_{m} q=0$. However, if condition (ii.) of Proposition 4 is satisfied, then $g \_$rank $\left(\operatorname{col}\left(C, A_{0}, A_{1}, \ldots, A_{m}\right)\right)=n$ and so there does not exist a vector $q \neq 0$ such that $C q=0$ and $A_{0} q=A_{1} q=\ldots=$ $A_{m} q=0$. Hence, for almost all the realizations of SBLS $\left(\Sigma_{\Lambda}\right)$, conditions of Proposition 4 are sufficient to ensure the observability.

In the case of system described in Example 3, Figure 2 represents $5 \mathrm{~A}$-disjoint edges extracted from digraph of Figure 1. Since all the state vertices are begin vertices of $\mathbf{Y}$-topped paths, this system is generically observable.

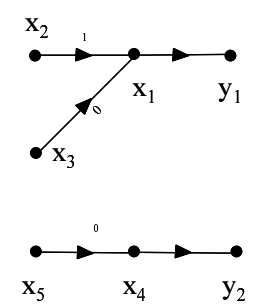

Figure 2. A set of 5 A-disjoint edges for example 3

As in [12] (Theorem 14.1) for the linear case, we can give simpler graphic conditions based on bipartite graphs:

Corollary 5 Structured bilinear system $\left(\Sigma_{\Lambda}\right)$ is generically observable iff in its associated digraph $\mathcal{G}\left(\Sigma_{\Lambda}\right)$

i. every state vertex is the begin vertex of an $\mathbf{Y}$-topped path; ii. g_rank $\left(\operatorname{col}\left(C, A_{0}, A_{1}, \ldots, A_{m}\right)\right)=n$ or equivalently, the maximal matching of the bipartite graph associated to matrix $\operatorname{col}\left(C, A_{0}, A_{1}, \ldots, A_{m}\right)$ is equal to $n$.

The main advantage of the previous corollary is its computational aspect. Indeed, the first condition can be checked using depth search algorithms. These algorithms have a complexity order $O(M \cdot N)$ where $M$ is the number of edges in the digraph and $N=n+p$ the number of vertices. For our digraphs, in the worst case $M=(m+1) \cdot n^{2}+n \cdot p$. Thus, the complexity of these algorithms, in our case, is $O\left(m \cdot n^{3}\right)$, (assuming without loss of generality that $p \leq n$ ). To check the second condition of Corollary 5 , we define the bipartite graph related to matrix $\operatorname{col}\left(C, A_{0}, A_{1}, \ldots, A_{m}\right)$ [12]. Then, we use the Bipmatch method [11], which allows to compute the cardinal of maximal matching into a bipartite graph. The complexity order of algorithms using this method is $O\left(M \cdot N^{0.5}\right)$, 
where, $M=(m+1) \cdot n^{2}+n \cdot p$ and $N=n+p+(m+1) \cdot n$. So, for checking the second condition of Corollary 5 we can use algorithms which have complexity order $O\left(m^{3 / 2} \cdot n^{5 / 2}\right)$. The proposed solution has an acceptable computational burden and so is very well suited to large scale systems.

The previous result can be seen as a generalization of the results concerning linear structured systems recalled in $[6,12]$ :

Proposition 6 A structured linear system $\left(\Sigma_{L, \Lambda}\right)$ : $\left\{\begin{array}{l}\dot{x}(t)=A x(t)+B u(t) \\ y(t)=C x(t)\end{array}\right.$ is generically observable iff in its associated graph $\mathcal{G}\left(\Sigma_{L, \Lambda}\right)$

i. every state vertex is the begin vertex of an $\mathbf{Y}$-topped path; ii. the maximal matching of the bipartite graph associated to matrix $\operatorname{col}(C, A)$ is equal to $n$.

The first condition of both Corollary 5 and Proposition 6 is exactly the same. Moreover, since for a linear system, there is no matrices $A_{i}, i=1, \ldots, m$, the second condition of Proposition 4 is equivalent to the second one of Corollary 5 in the linear case. Thus, in the linear case, Proposition 4 is equivalent to the one presented in [12] or to the one based on paths and cycles [6].

\section{Example}

In this section, we illustrate the results presented above with an example. This example is intentionally not very complicated. Nevertheless, it is clear that the observability criteria presented in Proposition 4 and Corollary 5 are very welladapted to more complex or large-scale systems using combinatorial programming techniques.

Consider the SBLS represented in the digraph of Figure 3. This system has 19 state components. The matrix representation of this system is not given because of lack of place. Nevertheless, it can be easily deduced from the digraph. Our aim is to show the simplicity of the proposed method on a relatively large example. The proposed conditions can be checked easily by hand whereas the rank test of the observability matrix is quite difficult to do by hand. In fact, this system is observable as each state vertex is a be-

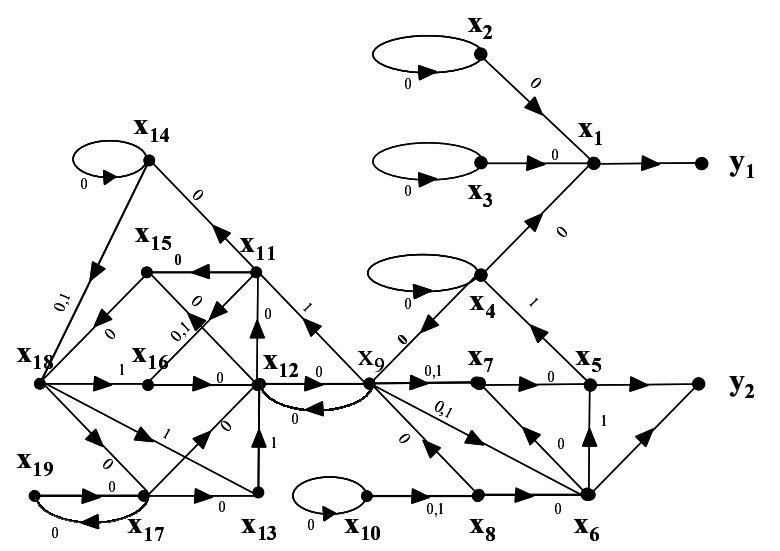

Figure 3. Illustrative example

gin vertex of an Y-topped paths and there exist 19 A-disjoint edges as it is displayed in Figure 4.

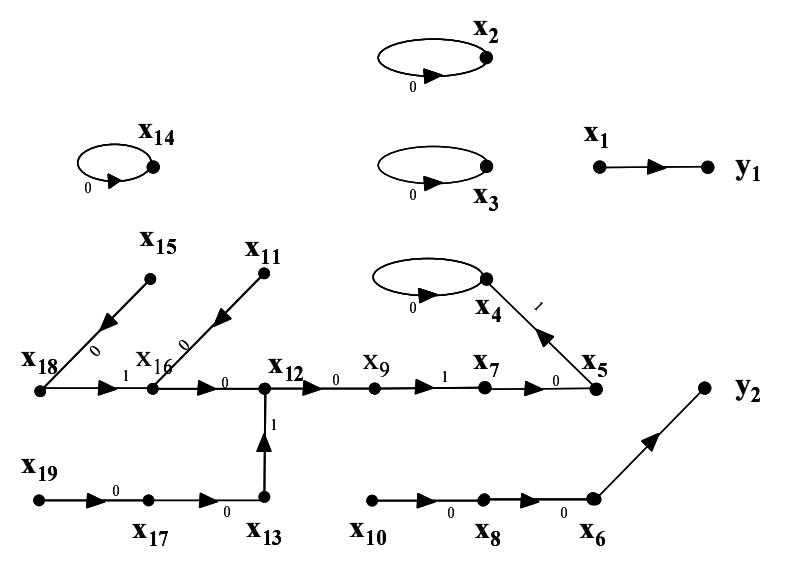

Figure 4. A set of 19 A-disjoint edges for the illustrative example

\section{Conclusion}

In this paper, we propose a new analysis tool to study the observability of structured bilinear systems. Using a new graph representation of this class of nonlinear systems, necessary and sufficient conditions for generic observability are given and expressed in graphic terms. These intrinsic conditions need few information about the system and are easy to check by means of combinatorial techniques or simply by hand for small systems. From a computational point of view, our approach is particularly suited for large-scale systems since it is free from numerical difficulties. Indeed, the proposed conditions can be easily implemented because they require simple computations based on finding edges in digraphs and on integer comparisons. Furthermore, the use of a graph-theoretic approach makes it easy to visualize the system structure. This may be very helpful for the optimisation of sensor placement to achieve the observability of the system. Indeed, starting from the above results, we can study, as in [3] for linear systems, the sensor location problem in order to achieve the generic observability.

\section{References}

[1] N. Andrë. Sparse Systems, Digraph Approach of Large-Scale Linear Systems Theory. TÜV, Köln, Germany, 1985.

[2] G. Bornard and H. Hammouri. A graph approach to uniform observability of linear multi output systems. In Proceedings of the $41^{\text {st }}$ IEEE Conference on Decision and Control, pages 701-706, Las Vegas, U.S.A., 2002.

[3] C. Commault, J-M. Dion, and D. H. Trinh. Observability recovering by additional sensor implementation in linear structured systems. In Proceedings of the $44^{\text {th }}$ IEEE Conference on Decision and Control, and the European Control Conference, Seville, Spain, 2005.

[4] H. D'Angelo. Linear time varying systems: Analysis and Synthesis. Allyn and Bacon, Boston, U.S.A., 1970.

[5] E. J. Davison and S. H. Wang. Properties of linear time-invariant multivariable systems subject to arbitrary output and state feedback. IEEE Transactions on Automatic Control, AC-18(1):24-32, 1973.

[6] J-M. Dion, C. Commault, and J. W. van der Woude. Generic properties and control of linear structured systems: a survey. Automatica, 39(7):1125-1144, 2003.

[7] O. M. Grasselli and A. Isidori. Deterministic state reconstruction and reachability of bilinear processes. In Proceedings of IEEE Joint Automatic Control Conference, pages 1423-1427, San Francisco, U.S.A., 1977. 
[8] T. Kailath. Linear systems. Prentice Hall Information and system science series. Prentice Hall, Englewood Cliffs, 1980.

[9] A. Kasinski and J. Lévine. A Fast Graph Theoretic Algorithm for the Feedback Decoupling Problem of Nonlinear Systems, volume 58, pages 550-562. Springer-Verlag, New York, P. A. Fuhrmann edition, 1984

[10] J. Lévine. A graph-theoretic approach to input output decoupling and linearization. In A. J. Fossard and D. Normand-Cyrot, editors, Nonlinear Systems, chapter 3, pages 77-91. Chapman \& Hall, London, U.K., 1997.

[11] S. Micali and V. V. Vazirani. An $O\left(\left|V^{1 / 2} E\right|\right)$ algorithm for finding maximum matching in general graphs. In Proceedings of the $21^{\text {st }}$ Annual Symposium on the Foundations of Computer Science, pages 17-27, 1980.

[12] K. Murota. System Analysis by Graphs and Matroids. SpringerVerlag, New York, U.S.A., 1987.

[13] K. J. Reinschke. Multivariable Control. A Graph Theoretic Approach. Springer-Verlag, New York, U.S.A., 1988.

[14] P. Sen. On the choice of input for observability in bilinear systems. IEEE Transactions on Automatic Control, AC-26(2):451-454, 1981.

[15] F. Svaricek. A graph theoretic approach for the investigation of the observability of bilinear systems. In Proceedings of the $12^{\text {th }}$ IFAC World Congress, pages 351-354, Sydney, Australia, 1993.

[16] J. W. van der Woude. A graph theoretic characterization for the rank of the transfer matrix of a structured system. Mathematics of Control, Signals and Systems, 4(1):33-40, 1991.

[17] J. W. van der Woude. The generic number of invariant zeros of a structured linear system. SIAM Journal of Control and Optimization, 38(1):1-21, 2000.

[18] D. Williamson. Observation of bilinear systems with application to biological control. Automatica, 13(3):243-254, 1977.

\section{Appendix A: Proof of Statement 1}

We prove hereafter that the maximal number of $A$-disjoint edges ending with vertices of $\mathbf{Y} \cup \mathbf{X}$ is equal to g_rank $(M)$, where $M=\operatorname{col}\left(C, A_{0}, A_{1}, \ldots, A_{m}\right)$.

To compute g_rank $(M)$, let us define bipartite graph $\mathcal{B}_{\mathcal{M}}=\left(\mathcal{V}^{+} ; \mathcal{V}^{-} ; \mathcal{E}_{\mathcal{M}}\right)$ associated to matrix $M$ and where $\mathcal{V}^{+} \stackrel{\text { def }}{=}\left\{\mathbf{x}_{\mathbf{1}}^{+}, \mathbf{x}_{\mathbf{2}}^{+}, \ldots, \mathbf{x}_{\mathbf{n}}^{+}\right\}, \mathcal{V}^{-} \stackrel{\text { def }}{=}$ $\left\{\mathbf{y}_{\mathbf{1}}^{-}, \mathbf{y}_{\mathbf{2}}^{-}, \ldots, \mathbf{y}_{\mathbf{p}}^{-}\right\} \cup\left\{\mathbf{x}_{\mathbf{0}, \mathbf{1}}^{-}, \mathbf{x}_{\mathbf{0}, \mathbf{2}}^{-}, \ldots, \mathbf{x}_{\mathbf{0}, \mathbf{n}}^{-}, \mathbf{x}_{\mathbf{1}, \mathbf{1}}^{-}, \mathbf{x}_{\mathbf{1}, \mathbf{2}}^{-}, \ldots, \mathbf{x}_{\mathbf{m}, \mathbf{n}}^{-}\right\}$ and $\mathcal{E}_{\mathcal{M}}=C^{M}$-edges $\cup\left(\bigcup_{k=0}^{m} A_{k}^{M}\right.$-edges $)$, with $C^{M}$-edges $\stackrel{\text { def }}{=}\left\{\left(\mathbf{x}_{\mathbf{i}}^{+}, \mathbf{y}_{\mathbf{j}}^{-}\right) \mid C(j, i) \neq 0\right\}$ and for $k=0, \ldots, m$, $A_{k}^{M}$ - edges $\stackrel{\text { def }}{=}\left\{\left(\mathbf{x}_{\ell}^{+}, \mathbf{x}_{\mathbf{k}, \mathbf{i}}^{-}\right) \mid A_{k}(i, \ell) \neq 0\right\}$.

Note that $C^{M}$-edges are the same than $C$-edges in $\mathcal{G}\left(\Sigma_{\Lambda}\right)$. From the results on the generic rank matrices [13], since all the parameters of matrices $A_{0}, \ldots, A_{m}$ and $C$ are assumed to be free, we have that $g_{-} \operatorname{rank}(M)$ is equal to the maximal number of disjoint edges in $\mathcal{B}_{\mathcal{M}}$, which is called the maximum matching in $\mathcal{B}_{\mathcal{M}}$.

If, for $i=1, \ldots, n$, vertices $\mathbf{x}_{\mathbf{0}, \mathbf{i}}^{-}, \mathbf{x}_{\mathbf{1}, \mathbf{i}}^{-}, \ldots, \mathbf{x}_{\mathbf{m}, \mathbf{i}}^{-}$are joined to form an unique vertex $\mathbf{x}_{\mathbf{i}}$ and if $\mathbf{x}_{\mathbf{i}}^{+}$and $\mathbf{y}_{\mathbf{i}}^{-}$are noted respectively $\mathbf{x}_{\mathbf{i}}$ and $\mathbf{y}_{\mathbf{i}}$, the number of disjoint edges in $\mathcal{B}_{\mathcal{M}}$ becomes the number of $A$-disjoint edges in $\mathcal{G}\left(\Sigma_{\Lambda}\right)$. Indeed, $k$ edges are disjoint in $\mathcal{B}_{\mathcal{M}}$ iff their corresponding edges in the original graph satisfy condition $\mathbf{C 1}$ (to have distinct begin vertices in $\mathcal{B}_{\mathcal{M}}$ ) and condition $\mathbf{C 2}$ (to have distinct end vertices in $\mathcal{B}_{\mathcal{M}}$ ).

Therefore, g_rank $(M)$ is equal to the maximal number of $A$ - disjoint edges ending with vertices in $\mathcal{G}\left(\Sigma_{\Lambda}\right)$ and Statement $\mathbf{1}$ is proved. 